\title{
Colonic Pseudo-obstruction as a Rare Complication of Pheochromocytoma
}

\author{
Kin Wai So, Hoi Ling Tsui, Kim Hung Tsang \\ Department of Radiology and Organ Imaging, United Christian Hospital, Hong Kong
}

Doi: 10.12890/2021_002421- European Journal of Case Reports in Internal Medicine - ๔ EFIM 2020

Received: 25/02/2021

Accepted: $11 / 03 / 2021$

Published: $26 / 03 / 2021$

How to cite this article: So KW, Tsui HL, Tsang KH. Colonic pseudo-obstruction as a rare complication of pheochromocytoma. EJCRIM 2021;8: doi:10.12890/2021_002421.

Conflicts of Interests: The Authors declare that there are no competing interests.

This article is licensed under a Commons Attribution Non-Commercial 4.0 License

\section{ABSTRACT}

Colonic pseudo-obstruction is characterized by dilatation of the colon without a structural lesion causing the obstruction. It usually involves the caecum and right side of the colon and is commonly observed in patients with severe illness or after surgery; it is rarely caused by pheochromocytoma.

The diagnosis of colonic pseudo-obstruction can be established by abdominal imaging including computed tomography (CT) of the abdomen or use of a water-soluble contrast enema. In additional to conservative or surgical treatment, alpha-blockers can be used in this setting to relieve the pseudo-obstruction.

\section{LEARNING POINTS}

- Colonic pseudo-obstruction is a rare manifestation of pheochromocytoma.

- A typical radiological feature of colonic pseudo-obstruction is the presence of a gradual transition at the splenic flexure of the colon.

- Alpha-blockers can be used in this setting to relieve pseudo-obstruction.

\section{KEYWORDS}

Pseudo-obstruction, pheochromocytoma, phenoxybenzamine

\section{CASE DESCRIPTION}

A 49-year-old Chinese woman with a history of left adrenalectomy for pheochromocytoma, was hospitalized due to abdominal distension and low back pain. Physical examination showed tachycardia (139 beats per minute), high blood pressure (162/98 mmHg), a distended abdomen and sluggish bowel motion but no peritoneal signs. Initial blood tests including a complete blood count, blood pH and base excess, liver and renal function tests were unremarkable. Abdominal radiography revealed a massively dilated colon and prominent loops of the small bowel, and expansile bone destruction at the right ilium (Fig. 1). Urgent computed tomography (CT) of the abdomen was performed for possible obstructing colonic lesions, which revealed a dilated proximal colon with a diameter measuring up to $9 \mathrm{~cm}$. There were no structural colonic lesions and smooth gradual tapering near the splenic flexure was observed (Fig. 2). There was no evidence of bowel compromise or perforation.

A diagnosis of colonic pseudo-obstruction was made based on the results of CT. The condition was initially managed conservatively. There was a significant increase in 24-hour urine normetanephrine (32,128 nmol/day, reference: <312). Phenoxybenzamine, propranolol and amlodipine were started for the treatment of sinus tachycardia and hypertension related to pheochromocytoma. In addition to normalization of blood pressure and heart rate, we also observed both clinical and radiological improvement of abdominal distension. 

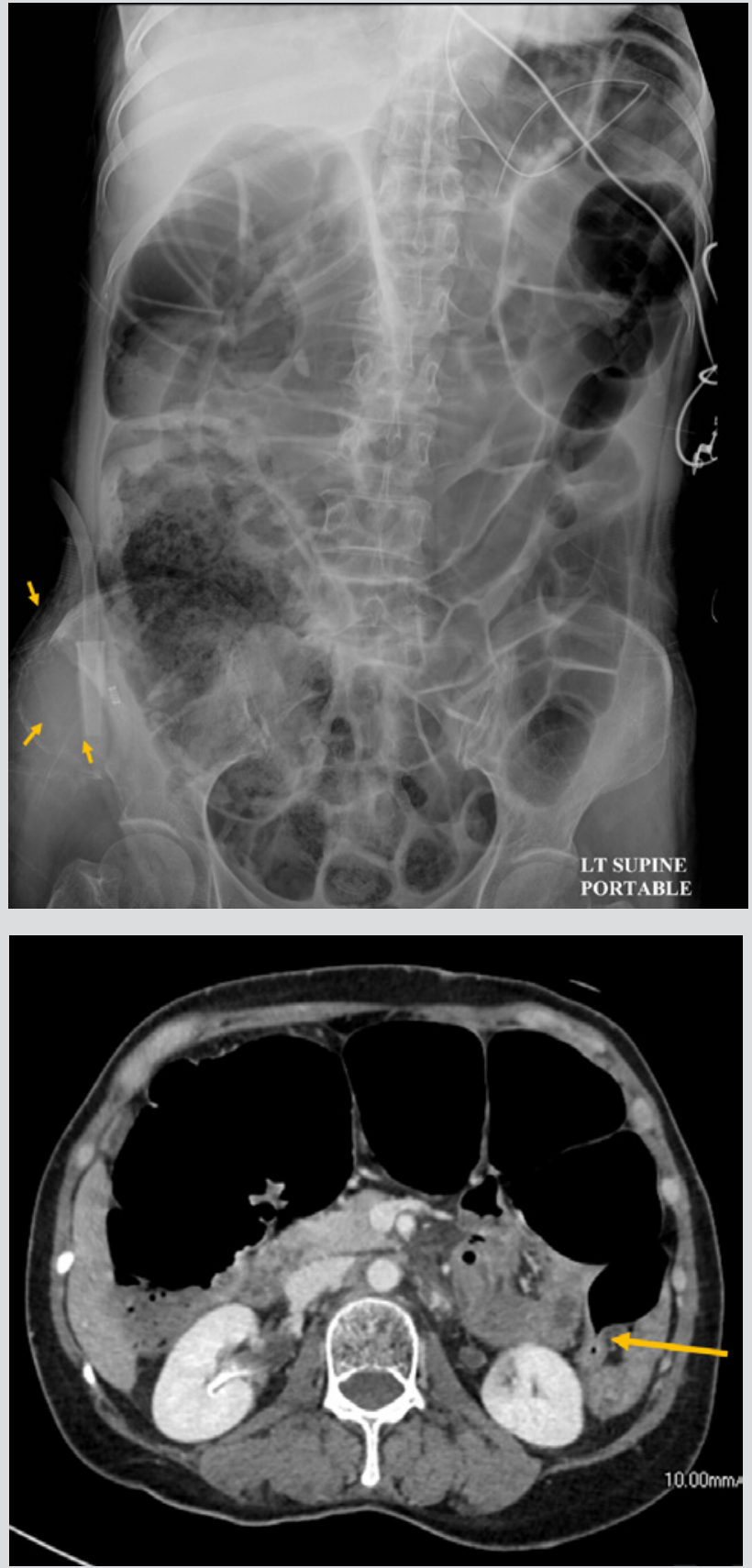

Figure 1. Abdominal radiography (AXR) demonstrated small and large bowel dilatation and expansile bone destruction at the right ilium (arrows)

Figure 2. Urgent abdominal computed tomography (CT) scan demonstrated intermediate transition at the splenic flexure (arrow) without an obstructing lesion

Serial follow-up abdominal radiographs taken 1 and 2 days after the administration of the alpha-blocker phenoxybenzamine showed improvement and gradual resolution of the pseudo-obstruction (Fig. 3). The transitional zone at the splenic flexure of the colon is clearly depicted in the abdominal radiograph taken 1 day after treatment (Fig. 3).

A technetium-99m bone scan was arranged and revealed multifocal increased radiotracer activity suggestive of bone metastases (Fig. 4) with increased uptake over the left femoral and right iliac regions. The patient underwent prophylactic long nail fixation due to left femoral bone destruction. Metastatic pheochromocytoma was confirmed in the surgical samples of the left femur. 


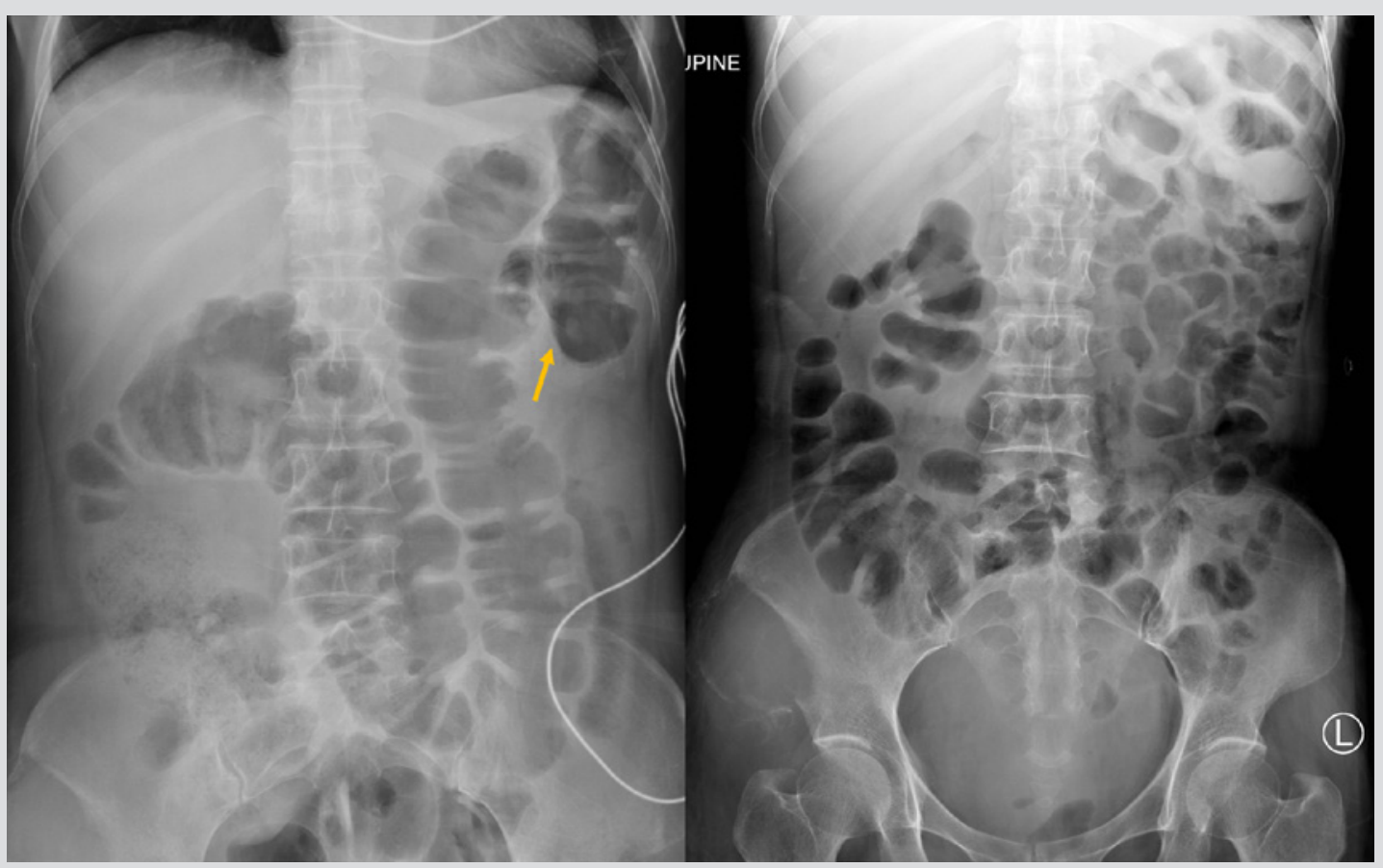

Figure 3. Abdominal radiography (AXR) taken 1 and 2 days after the administration of phenoxybenzamine showed improvement and gradual resolution of the pseudo-obstruction, with the transition zone (arrow) clearly depicted in the left image

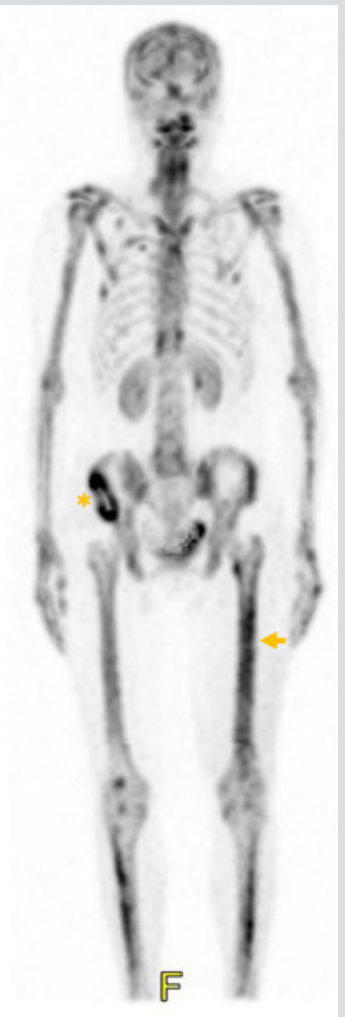

Figure 4. A bone scan showed multifocal radiotracer activity especially at the left femur (arrow) and right ilium (asterisk)

\section{DISCUSSION}

Acute pseudo-obstruction of the colon, which is also known as Ogilvie's syndrome, is characterized by dilatation of the colon without an anatomical lesion, causing bowel lumen obstruction. It usually involves the caecum and right hemicolon.

According to a retrospective study of a case series of 400 patients by Vanek et al. ${ }^{[1]}$, abdominal distension is the characteristic presenting feature of acute colonic pseudo-obstruction. Some $83 \%$ of patients have associated abdominal pain, while nausea and vomiting can be seen 
in up to $60 \%$ of patients. Approximately $10 \%$ of patients present with absence of bowel sounds. Fever and abdominal tenderness are more commonly observed in cases complicated with colonic perforation. Acute colonic pseudo-obstruction is mostly seen in hospitalized patients following surgery or severe systemic illness. The most common predisposing factors are non-operative trauma, infection and heart disease, each contributing to $10-11.3 \%$ of cases.

Acute colonic pseudo-obstruction has been described as a rare complication of pheochromocytoma in several case reports ${ }^{[2,3]}$. Although the exact underlying mechanism remains unclear, it may be related to high levels of circulating catecholamines resulting in decreased intestinal peristalsis, motility and tone ${ }^{[2]}$. There are two plexuses in the enteric nervous system: an outer myenteric plexus and an inner submucosal plexus. Gastrointestinal movements are regulated by the outer myenteric plexus which is modulated by the parasympathetic and sympathetic nervous systems. The sympathetic nerve endings release norepinephrine, which inhibits the enteric plexus through activation of the $\alpha 1-, \alpha 2$ - and $\beta 2$-adrenergic receptors, resulting in a reduction in bowel movement, gastrointestinal secretion and blood flow $^{[3]}$. Circulating epinephrine may cause mesenteric vasoconstriction leading to ischaemia and inhibit gastrointestinal peristaltic activity through stimulation of $\alpha 1 / \alpha 2$ and $\beta 2$ receptors, respectively ${ }^{[3]}$.

Acute colonic pseudo-obstruction should be suspected in patients with the above risk factors who present with abdominal distension and hyper-resonant percussion. Abdominal radiography and CT of the abdomen should be performed to establish the diagnosis and to rule out structural causes. Colonic pseudo-obstruction typically displays an intermediate transitional zone at or adjacent to the splenic flexure [4], and such radiological findings have also been shown in our case (Figs. 2 and 3). These radiographic findings should alert the clinicians to this diagnosis. A water-soluble contrast enema can also be used to establish the diagnosis by demonstrating colonic dilatation and the absence of a structural lesion causing obstruction. However, it has become less popular than CT of the abdomen. Colonoscopy should not be considered to establish the diagnosis as it could further inflate the colon.

The risk of perforation increases when the caecal diameter exceeds $10-12 \mathrm{~cm}$ or colonic distension persists for 6 or more days ${ }^{[5]}$. The goal of treatment of colonic pseudo-obstruction is to minimize the risk of colonic perforation and ischaemia by decompression of the colon. In the absence of severe abdominal pain, colonic dilation $>12 \mathrm{~cm}$ or symptoms of peritonitis, initial treatment can be conservative ${ }^{[6]}$. Neostigmine or colonoscopy decompression could be considered in patients who are not suitable for or have failed conservative treatment ${ }^{[6]}$. In our case, initial management for pseudo-obstruction was conservative, but we observed an improvement in 2 days following the administration of an alpha-blocker which was intended for the treatment of sinus tachycardia and hypertension. Clinical experience with alpha-blockers in the management of pheochromocytoma/paraganglioma-associated pseudo-obstruction is limited due to the low incidence, as reflected by a total number of only 34 case reports despite a comprehensive literature search by Osinga et al. ${ }^{[3]}$. In this literature review, the intravenous administration of phentolamine, a competitive $\alpha 1$ - and $\alpha 2$-adrenergic receptor antagonist, was the most frequently applied pharmacological treatment (78\%) and the majority of patients showed clinical improvement. When standard management for pseudo-obstruction fails, the use of an alpha-blocker can be considered in this setting.

Prognosis depends on caecal diameter, duration of dilatation, and bowel status. The mortality rate is approximately $15 \%$ with early appropriate management compared with approximately $40 \%$ in cases of perforated or ischaemic bowel.

Our case shows the typical radiographic findings of colonic pseudo-obstruction, and pheochromocytoma as a rare cause of it. Early recognition of pseudo-obstruction is important for the initiation of prompt treatment and prevention of lethal complications.

\section{REFERENCES}

1. Vanek VW, Al-Salti M. Acute pseudo-obstruction of the colon (Ogilvie's syndrome). An analysis of 400 cases. Dis Colon Rectum 1986;29(3):203-210.

2. Sweeney AT, Malabanan AO, Blake MA, de las Morenas A, Cachecho R, Melby JC. Megacolon as the presenting feature in pheochromocytoma. J Clin Endocrinol Metab 2000;85(11):3968-3972.

3. Osinga TE, Kerstens MN, van der Klauw MM, Koornstra JJ, Wolffenbuttel BHR, Links TP, et al. Intestinal pseudo-obstruction as a complication of paragangliomas: case report and literature review. Neth J Med 2013;71(10):512-517.

4. Choi JS, Lim JS, Kim H, Choi J-Y, Kim M-J, Kim NK, et al. Colonic pseudoobstruction: CT findings. AJR Am J Roentgenol 2008;190(6):1521-1526.

5. Saunders MD. Acute colonic pseudo-obstruction. Best Pract Res Clin Gastroenterol 2007;21(4):671-687.

6. Naveed M, Jamil LH, Fujii-Lau LL, Al-Haddad M, Buxbaum JL, Fishman DS, et al. American Society for Gastrointestinal Endoscopy guideline on the role of endoscopy in the management of acute colonic pseudo-obstruction and colonic volvulus. Gastrointest Endosc 2020;91(2):228-235. 